\title{
Research on Elevator Energy Feedback based on Active Inverter Zeyu $\mathrm{He}^{\mathrm{a}}$, Jinhu Liao ${ }^{\mathrm{b}}$ \\ School of Automation, Wuhan University of Technology, Wuhan 430070, China \\ ahezeyu@whut.edu.cn, b1296584600@qq.com
}

Keywords: elevator, energy feedback, inverter, SVPWM

\begin{abstract}
Aiming at reducing the elevator's energy consumption, this paper analyses the energy feedback technology, and designs an energy feedback system used for elevator which can return the extra energy to the power grid in the generating state of the motor. The system adopts active inverter technology to achieve energy recycle. In this system, double closed-loop control is used to stabilize the input voltage and realize unity power factor operation. A simulation model is built to demonstrate the performance of the system based on Simulink/Matlab.
\end{abstract}

\section{Introduction}

Elevator is widely popularized with the development of society. As high-energy equipment, its consumption holds a large proportion of the whole building's power consumption. The research of saving energy on elevator is pressing in this time of energy shortage.

Elevator drives potential energy load. The load consists of the car and the counterweight and has a balance coefficient of nearly 0.5 . Thus when the elevator is rising with heavy load or descending with light load, the motor works in the electro-motion state; and on the contrary condition, the motor works in the generating state. Elevators usually use VVVF converter to drive three phase synchronous motor. Due to the unidirectional continuity of the diodes which composed the three-phase pre-rectifier, traditional VVVF elevator controllers cannot transfer the energy to the power grid ${ }^{[1]}$. The generating process of the motor will produce pump voltage and raise the voltage of the energy storage capacitor. Traditional elevators usually use resistance braking to reduce the voltage on the DC bus. According to research, $25 \%$ to $30 \%$ of the energy is consumed by the resistor ${ }^{[2]}$.

Energy feedback technology has a vast application prospect. In some foreign countries, many advanced energy feedback devices are designed to use the extra energy and return it to the power grid. But these products have high cost and high demand of the power grid. In China, the elevator energy feedback system hasn't got good promotion. Thus this paper studies the related technology and designs an elevator energy feedback system which has low cost and simple installation.

\section{Design of the Energy Feedback System}

For recycling the extra energy of the elevator, this paper designs an energy feedback system which is showed in Fig.1. The key of the energy feedback is active inverter ${ }^{[3]}$. When the motor is working in the generating state, the extra energy can be saved by transforming the DC power into three-phase AC power and returning it to the power grid. The system adopts DSP chip as the central control unit, and is mainly made up by control circuit, inverter circuit and sync circuit. In working condition of the system, the electric energy on the DC bus is returned to the power grid to decrease the voltage on the DC bus, and this process realizes energy regeneration. The system is independent to the elevator controller and few work need to be done to install the equipment. 


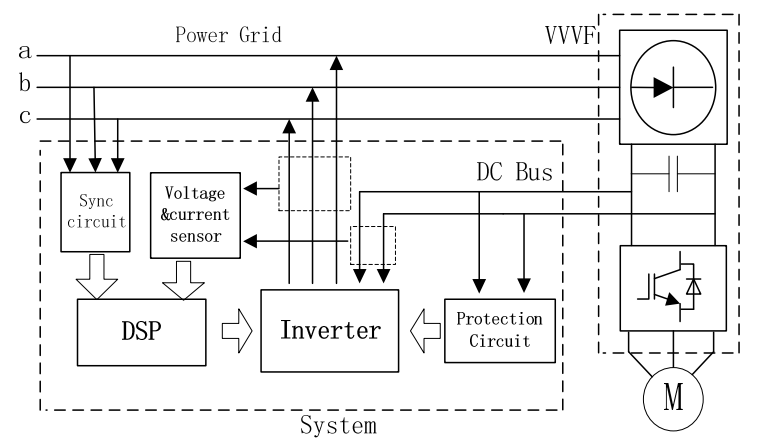

Fig.1 Elevator energy feedback system

The three-phase full-bridge inverter is the core of the feedback device, reversing the regenerated power from the DC bus to the power grid ${ }^{[4]}$. Fig. 2 shows the inverter circuit constructed by six IGBTs. As the central switching element of the inverter, IGBT has good electrical performance, like high switching frequency, large drive current, low wastage and so on. IGBT elements are controlled by the PWM signal from the DSP chip.

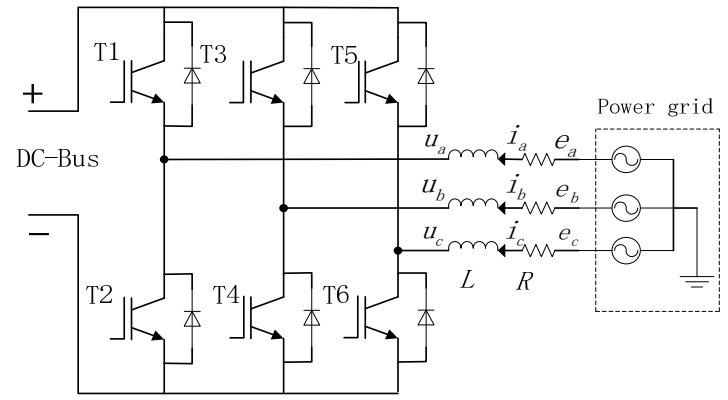

Fig.2 Power inverter circuit

The system adopts TMS320LF2407A as the central controller. This control chip has high cost performance and is able to meet demand of the system. TMS320LF2407 family has a working frequency of $30 \mathrm{MHz}$ and strong functions of signal processing and data calculating. The chip has abundant resources including 2 event management modules, 2 general timers of 16 bit, 8 PWM channels, $16 \mathrm{AD}$ channels and so on. Both voltage and current of the system are measured by hall sensors and then sampled by AD transform. In working process, the DSP chip gets sampled values in real time, makes calculation of coordinate transform and closed-loop control and generates six PWM pulses through the PWM unit based on SVPWM.

\section{The method of control}

Besides returning the extra energy to the power grid, the system is supposed to realize the functions of stabilizing the voltage of the DC bus and adjusting the power factor. To achieve these functions, math model of the system is built. The equation of the inverter side is showed in Eq.1.

$$
\left\{\begin{array}{l}
L \frac{d i_{a}}{d t}=e_{a}-R i_{a}-u_{a} \\
L \frac{d i_{b}}{d t}=e_{b}-R i_{b}-u_{b} \\
L \frac{d i_{c}}{d t}=e_{c}-R i_{c}-u_{c}
\end{array}\right.
$$

The mathematical model in the three-phase static coordinate system is difficult to analyze and control. For simplifying the calculation, coordinate transform method is used. From the three-phase static frame to the $\mathrm{d}-\mathrm{p}$ rotating frame, three-phase $\mathrm{AC}$ values are transformed into DC values by $3 \mathrm{~s} / 2 \mathrm{r}$ coordinate transform which is showed in Fig.3 (a). In stable working state, both d-axis component and q-axis component are constant. In the $d-p$ frame, vector of the grid-side voltage is set to coincide with d-axis. By this way d-axis and q-axis component can separately represent active and reactive power. This coordinate transform realizes the independent control of active and reactive power. 


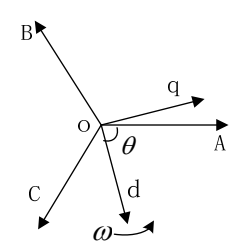

(a) $3 \mathrm{~s}$ and $2 \mathrm{r}$

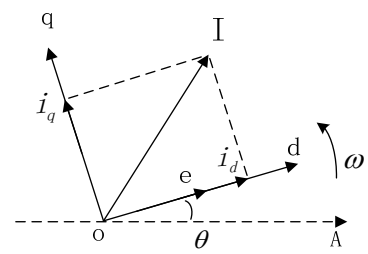

(b) Diagram of current and

Fig.3 Diagram of the $3 \mathrm{~s} / 2 \mathrm{r}$ transform

The rule of the $3 \mathrm{~s} / 2 \mathrm{r}$ transform satisfies Eq.2.

$$
\left[\begin{array}{l}
u_{d} \\
u_{q}
\end{array}\right]=\sqrt{\frac{2}{3}} *\left[\begin{array}{ccc}
\cos \theta & \cos \left(\theta-120^{\circ}\right) & \cos \left(\theta+120^{\circ}\right) \\
-\sin \theta & -\sin \left(\theta-120^{\circ}\right) & -\sin \left(\theta+120^{\circ}\right)
\end{array}\right] *\left[\begin{array}{l}
u_{a} \\
u_{b} \\
u_{c}
\end{array}\right]
$$

In Eq.2, $\theta=\theta_{0}+\omega \mathrm{t}$. $\theta_{0}$ is the initial included angle between $\mathrm{d}$-axis and A-axis. Both the voltage and the current of the grid side are transformed into DC signal. Phase diagram of current and grid-side voltage is showed in Fig.3 (b).

The system use double closed-loop control method. With $3 \mathrm{~s} / 2 \mathrm{r}$ coordinate transform, the relation of the values in stable state is showed in Eq.3.

$$
\left\{\begin{array}{l}
u_{d}=e_{d}-R i_{d}+\omega L i_{q} \\
u_{q}=e_{q}-R i_{q}-\omega L i_{d}
\end{array}\right.
$$

$e_{d}, e_{q}, i_{d}, i_{q}$ are power source voltage vectors and current vectors in d-q vector space. $\omega$ stands for the angular frequency of the grid ${ }^{[5]}$. For $i_{d}$ and $i_{q}$ are coupled with each other, it is difficult to control each variable separately. Using the feed-forward control strategy, we are able to decouple those variables, and Eq.3 is transformed into two independent SISO processes ${ }^{[6]}$. The diagram of the double-loop control is showed in Fig.4. The DC bus voltage loop is the outer loop, and the grid side current loop is the inner loop.

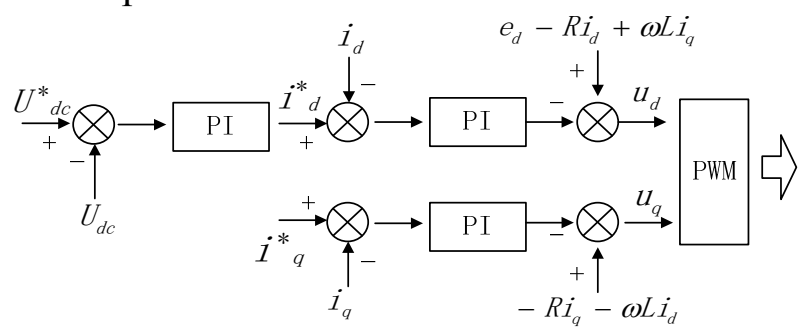

Fig.4 Block diagram of double-loop control

The system adopts PI controller to realize closed-loop control. After the feed-forward decoupling, $i_{d}$ loop is used to control the active power and the voltage of the DC bus, and $i_{q}$ loop is used to control the reactive power. $\mathrm{U}_{\mathrm{dc}}{ }^{*}$ is voltage command of the DC bus which is set to $620 \mathrm{~V} . \mathrm{i}_{\mathrm{d}}{ }^{*}$ and $\mathrm{i}_{\mathrm{q}}{ }^{*}$ are current commands for active current and reactive current respectively. $\mathrm{i}_{\mathrm{q}}{ }^{*}$ is set to zero to eliminate reactive power and realize unity power factor.

Space vector pulse width modulation (SVPWM) is used in this system to control the inverter and its diagram is showed in Fig.5.

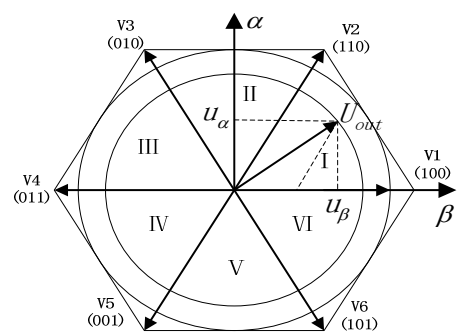

Fig.5 Diagram of SVPWM

This procedure needs the instruction of the AC side voltage vector in $\alpha-\beta$ rotary coordinate system. The output value of the double-closed loop control is $u_{d}$ and $u_{\mathrm{q}}$ which are transformed from three-phase AC signals by $3 \mathrm{~s} / 2 \mathrm{r}$ transform. So the inverse Park transformation is needed to transform $\mathrm{u}_{\mathrm{d}}$ and $\mathrm{u}_{\mathrm{q}}$ into $\mathrm{u}_{\alpha}$ and $\mathrm{u}_{\beta}$, and its expression is showed in Eq.4. 
$\left[\begin{array}{l}u_{\alpha} \\ u_{\beta}\end{array}\right]=\left[\begin{array}{cc}\cos \theta & -\sin \theta \\ \sin \theta & \cos \theta\end{array}\right] *\left[\begin{array}{l}u_{d} \\ u_{q}\end{array}\right]$

In order to quickly figure out which section the $\mathrm{AC}$ side voltage vector is in, three parameters $\mathrm{A}$, $\mathrm{B}, \mathrm{C}$ are appointed: if $u_{\beta}>0, \mathrm{~A}=1$, otherwise $\mathrm{A}=0$; if $\frac{\sqrt{3}}{2} u_{\alpha}-\frac{1}{2} u_{\beta}>0, \mathrm{~B}=1$, otherwise $\mathrm{B}=0$; if $-\frac{\sqrt{3}}{2} u_{\alpha}-\frac{1}{2} u_{\beta}>0, \mathrm{C}=1$, otherwise $\mathrm{C}=0^{[7]}$; And the number of the section is:

$\mathrm{N}=\mathrm{A}+2 * \mathrm{~B}+4 * \mathrm{C}$

Then, the switch period of the two voltage vectors in the section of the AC side voltage vector can be calculated by vector resolution method to produce the PWM control signal.

\section{Simulation and Analysis}

In order to demonstrate the feasibility of the system, the model of the system is built in Simulink of Matlab. The simulation model of the energy feed back system is depicted in Fig.6, and the simulation parameter settings are showed in Tab.1. The system of Simulink is made up by the power grid, sync module, SVPWM module, inverter module, and closed-loop controller.

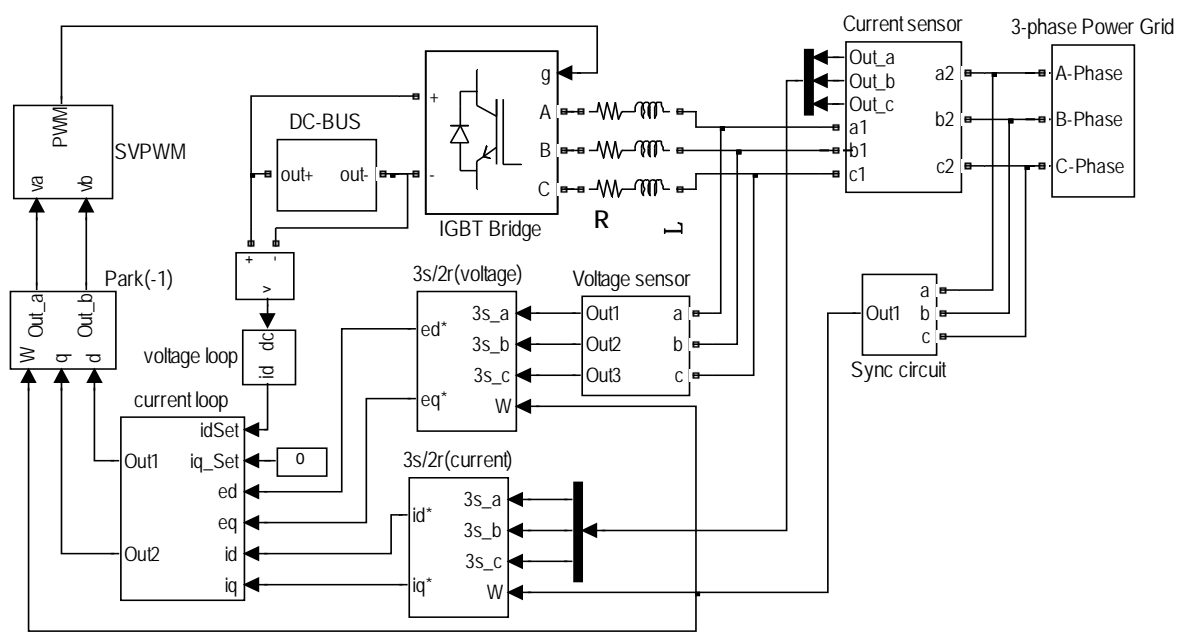

Fig.6 System structure of the Simulink software

Table 1 Simulation parameter settings

\begin{tabular}{|l|c|}
\hline Switching frequency & $5 \mathrm{KHz}$ \\
\hline Inductance( $\mathrm{L})$ & $5 \mathrm{mh}$ \\
\hline Resistance $(\mathrm{R})$ & $0.5 \Omega$ \\
\hline Max type size & $4 \mathrm{e}-6$ \\
\hline Relative tolerance & $1 \mathrm{e}-3$ \\
\hline Algorithm & Ode $23 \mathrm{tb}$ \\
\hline $\mathrm{K}_{\mathrm{p}} / \mathrm{K}_{\mathrm{i}}\left(\mathrm{i}_{\mathrm{d}}\right.$ loop) & $2.5 / 4.5$ \\
\hline $\mathrm{K}_{\mathrm{p}} / \mathrm{K}_{\mathrm{i}}\left(\mathrm{i}_{\mathrm{q}}\right.$ loop) & $3.5 / 4.5$ \\
\hline $\mathrm{K}_{\mathrm{p}} / \mathrm{K}_{\mathrm{i}}$ (voltage loop) & $1.6 / 3.5$ \\
\hline
\end{tabular}

Set the initial voltage of the DC bus to $650 \mathrm{~V}$ and start the simulation. The output current waveform of the system in stable working state is depicted in Fig.7. The simulation results show that the system can produce output current whose waveform is approximate sine wave. And the A-phase output current is out of phase with A-phase grid side voltage, which means the system is in unity power factor operation. 


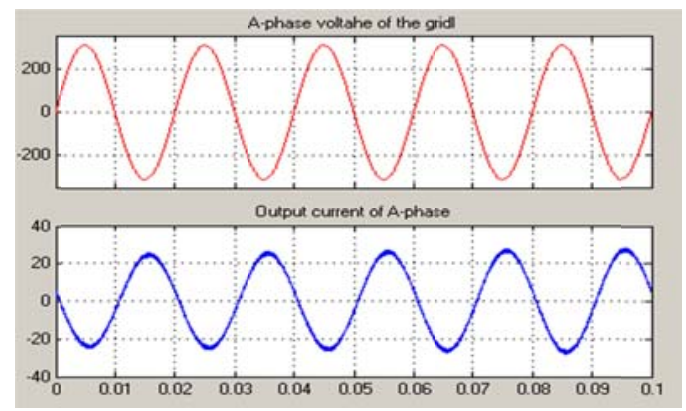

Fig.7 A-phase voltage and current of the grid side

Use FFT to analyze the current signal in Fig.7 from $0.1 \mathrm{~s}$ to $0.16 \mathrm{~s}$, and we can get the A-phase current harmonic analysis, which is showed in Fig.8. From the results of the analysis, the THD (Total Harmonic Distortion) of the signal is only $1.25 \%$, which is totally meeting the national standard about harmonic component need to be less than $5 \%{ }^{[8]}$.

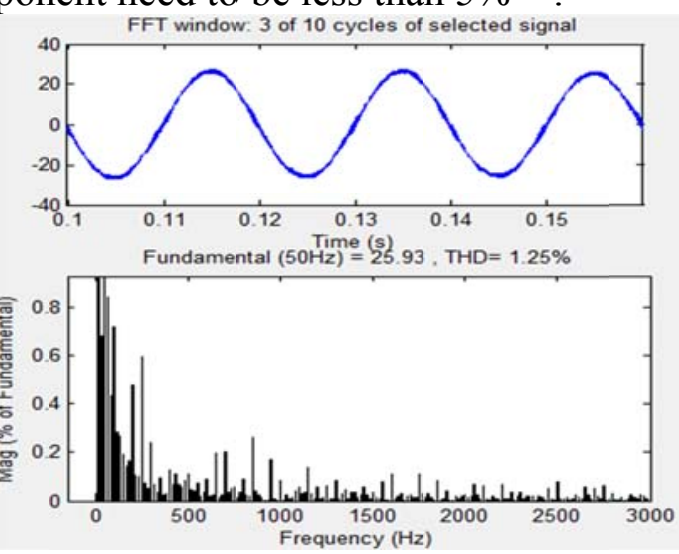

Fig.8 Output current and its FFT analysis

The system uses double closed-loop control to stabilize the voltage of the DC bus. In the simulation, start the system at $0.02 \mathrm{~s}$ when the real voltage of the DC bus is up to $650 \mathrm{~V}$ on condition that the set voltage is $620 \mathrm{~V}$, and the voltage waveform of the DC bus is showed in Fig.9 (a). The voltage of the DC bus stabilizes at the set value within $0.03 \mathrm{~s}$. The step response of the system about the set voltage is showed in Fig.9 (b). After the set voltage changing from $620 \mathrm{~V}$ to $640 \mathrm{~V}$ at $0.02 \mathrm{~s}$, the voltage of the DC bus stabilizes at the new set value within $0.02 \mathrm{~s}$.
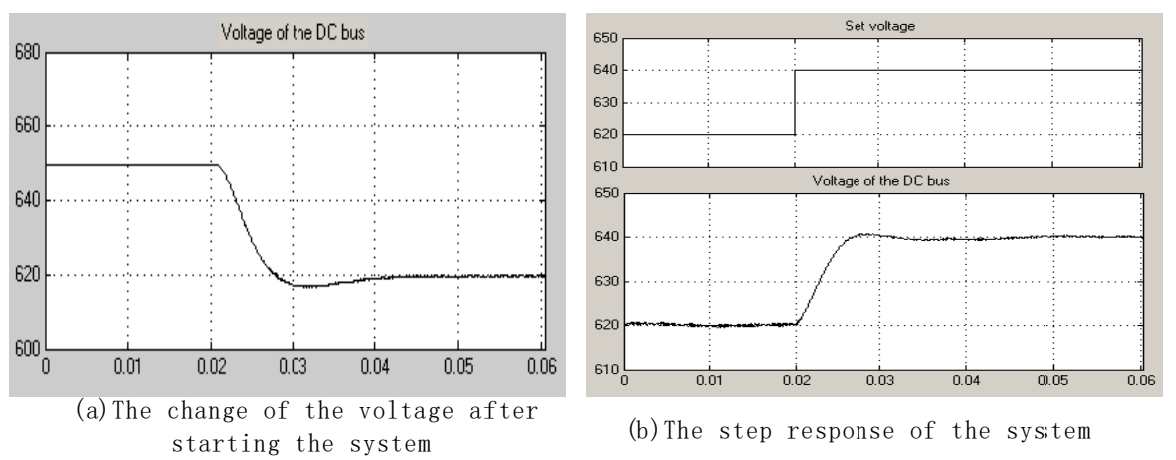

Fig.9 Voltage waveform of the DC bus

The simulation results show that the energy feedback system can recycle the extra energy, stabilize the voltage of the DC bus and realize unity power factor operation. The output power of the system has high quality and small interference on the power grid. The performance of the system can well meet the need of the elevator energy feedback.

\section{Conclusion}

The elevator energy feedback system designed in this paper achieves the aim of returning the extra electoral energy from the DC bus to the power grid effectively in motor's generating state. And the simulation results show that the proposed method has good predictive performance and is feasible for the elevator energy feedback system. The system could stabilize the voltage of the DC 
bus, and the output electrical energy from the system has power factor of nearly 1 and small interference on the power grid. The system has obvious efficient of saving energy and is suitable to be popularized.

\section{References}

$[1,5,7]$ Yiqiu Liu, Xiaohong Wang, Lianfang Tian. Research of elevator energy feedback system based on converters using SVPWM. Transportation, Mechanical, and Electrical Engineering Conference. Changchun, Dec.2011, p. 998-1002

[2] Chunyu Zhang: Design and Research of Grid Connected System of Elevator Energy Feedback (MA.Eng, Dalian University of Technology, China 2014).p. 2.

[3] Jishen Peng, Weiwei Wang, Liye Song. A New Elevator Control System of Energy Feedback and Network Design. Computer Systems \& Applications. Vol. 21(2012), No.03,p. 80-82

[4] Jinping He, Chengxiong Mao, Jiming Lu, Jiawei Yang. Design and Implementation of an Energy Feedback Digital Device Used in Elevator. IEEE Transactions on Industrial Electronics. Wuhan,Oct.2011, p. 4636-4642

[6] J. Rodríguez, J. Dixon, J. Espinoza, P. Lezana. PWM Regenerative Rectifiers: State of the Art. IEEE Transactions on Industrial Electronics. Vol.52( 2005), No.01,p. 5-22.

[8] Rui Wang, Fei Luo, Xiaohong Huang, Peng Li. New elevator energy feedback control system design based on fuzzy PID controller. Power Engineering and Automation Conference. Wuhan, Sep. 2012, p.1-6 